\title{
Sexualité, mariage et esclavage au Soudan français à la fin du XIX ${ }^{e}$ siècle
}

Sexuality, marriage and slavery in the French Soudan at the end of the $19^{\text {th }}$ century

\section{Marie Rodet}

\section{(2) OpenEdition}

\section{Journals}

\section{Édition électronique}

URL : https://journals.openedition.org/clio/10009

DOI : 10.4000/clio.10009

ISSN : 1777-5299

\section{Éditeur}

Belin

Édition imprimée

Date de publication : 1 mai 2011

Pagination : 45-64

ISBN : 978-2-8107-0157-5

ISSN : $1252-7017$

\section{Référence électronique}

Marie Rodet, « Sexualité, mariage et esclavage au Soudan français à la fin du XIXe siècle », Clio.

Femmes, Genre, Histoire [En ligne], 33 | 2011, mis en ligne le 01 mai 2013, consulté le 27 avril 2022.

URL : http://journals.openedition.org/clio/10009; DOI : https://doi.org/10.4000/clio.10009 


\section{Sexualité, mariage et esclavage au Soudan français à la fin du XIX ${ }^{\mathrm{e}}$ siècle ${ }^{1}$}

Marie RODET

En avril 1904, une certaine Yackaré s'adresse au commandant du cercle de Kayes ${ }^{2}$ pour réclamer sa fille, Gassa, abandonnée par un adjudant français à Nioro suite au rapatriement de ce dernier en France: «J'ai l'honneur de vous faire une plainte contre un adjudant qui m'a pris ma fille comme fiancée $»^{3}$. L'administration entreprend alors une enquête qui révèle que Gassa n'est pas la fille mais l'ancienne esclave de Yackaré. L'adjudant a en effet demandé deux

1 Les recherches pour cet article ont été rendues possibles grâce à une allocation post-doctorale Île-de-France de l'Institut Émilie du Châtelet en 2008 et à un séjour comme chercheuse invitée au Center for African Studies de l'Université de Stanford en 2010. Je remercie Pascale Barthélémy, Boris Lesueur, Sarah Zimmermann, de même que les lecteurs anonymes de Clio pour leurs commentaires judicieux. Cet article a également bénéficié de nombreux échanges sur les questions de genre, sexualité, mariage, colonialisme et esclavage en Afrique lors des conférences et séminaires auxquels j'ai pu participer entre 2007 et 2010 en Amérique du Nord et en Europe, notamment avec Emily Burrill, Odile Goerg, Martin Klein, Ben Lawrance, Richard Roberts et Elizabeth Thornberry.

2 Kayes est la première capitale du Soudan français (qui deviendra la République du Mali en 1960), une des colonies d'Afrique occidentale française. Les colonies sont administrées par un gouverneur et divisées administrativement en cercles. Les cercles sont gérés par des « commandants » qui concentrent l'ensemble des pouvoirs administratifs, de police, de magistrat, de procureur et de geôlier. La plupart des premiers administrateurs des cercles étant des militaires de la conquête, le terme « commandant » est entré dans l'usage courant.

3 Archives nationales du Mali (ANM), Koulouba, fonds ancien (FA), 1 E 212 : Correspondance affaires politiques du cercle de Nioro, 1902-1911. 
ans auparavant à Yackaré de lui racheter la jeune fille pour en faire sa concubine. Cette dernière, après avoir refusé, a finalement consenti à la lui donner en mariage contre la somme de 40 francs. Gassa, une fois mariée, réclame un certificat de liberté auprès de l'administration, qu'elle obtient en juillet 1902. Elle vit deux ans avec l'adjudant français puis, suite au départ de celui-ci, refuse de retourner chez son ancienne maîtresse, d'où la plainte déposée par celle-ci.

Cet échange de correspondances ne révèle sans doute qu'une infime partie de la complexité des relations intimes nouées entre hommes européens et femmes africaines dans les premières années de la colonisation au Soudan français. Si les mémoires des anciens militaires ayant participé à la conquête et des premiers administrateurs coloniaux confirment que le fait de prendre comme concubine une femme locale, souvent une esclave, était chose courante au tournant $\mathrm{du} \mathrm{XX}^{\mathrm{e}}$ siècle $^{4}$, peu de documents produits par l'administration coloniale attestent de ces pratiques ${ }^{5}$.

Cet article analyse quatre affaires qui eurent lieu entre 1902 et 1906 dans la région de Kayes, et qui montrent l'importance de la sexualité et du couple comme lieux où se jouent, à l'époque de la fin de l'esclavage, de complexes rapports de pouvoir ${ }^{6}$. Ces affaires permettent en particulier d'identifier la fabrique genrée au quotidien de catégories telles que «colonisateurs » et «colonisés » et de mesurer leur impact sur la politique coloniale d'émancipation des femmes esclaves. Elles dévoilent également les contestations et les reconfigurations de ces catégories par des acteurs et actrices

4 Simonis 1993, 2007.

5 Deux notables exceptions cependant: le scandale des esclaves du capitaine Quiquandon et l'arrestation du lieutenant Mangin qui partageait avec ses subordonnés les femmes prises pendant les sièges (Kanya-Forstner 1969 : 228). Si ces dernières affaires s'avèrent sans doute des cas extrêmes (Tymowski 2000), les recherches de Simonis (1993 et 2007) montrent précisément l'ampleur des pratiques de concubinage à cette époque, même s'il n'aborde pas directement la question du statut des compagnes des militaires et autres «broussards ». La situation des femmes des tirailleurs sénégalais a été plus amplement étudiée. Voir notamment Capitaine Marceau 1911, Echenberg 1980, Thompson 1990, Mann 2006, Duparc 2009, Zimmerman (à paraître).

6 Goerg 2007: 7. 
finalement peu étudié-e-s dans l'histoire du Mali : les femmes esclaves et les agents subalternes de la colonisation. On ne sait malheureusement par les archives que peu de choses sur ces protagonistes, ni leur âge, ni leur origine notamment. L'étude de ces affaires permet cependant de mieux cerner les contours complexes et peu connus des relations intimes qui, si elles n'excluent pas toujours l'affectif et l'assentiment, n'en sont pas moins établies dans des contextes spécifiques de pouvoir et de violence. Mais c'est aussi dans ces contextes que certaines femmes esclaves réussissent à obtenir leur liberté ${ }^{7}$.

\section{« Ce n'était pas une captive qu'il entendait acheter, mais une femme »}

Les premières mesures prises par les Français pour limiter l'esclavage dans la région de Kayes datent de 1895. Le gouverneur Grodet décide alors l'interdiction de la traite sur les territoires nouvellement conquis. Les caravanes traversant la région sont à partir de cette date régulièrement interceptées par l'administration et leurs esclaves envoyé-e-s dans les villages de liberté où, après trois mois de séjour, ils/elles peuvent obtenir un certificat de liberté. Lorsque William Ponty devient délégué du gouverneur général à Kayes en 1899, la politique abolitionniste dans la région prend un nouvel élan. En 1901, une circulaire interdit que les esclaves résidant dans les villages de liberté soient rendu-e-s à leurs propriétaires comme cela se faisait jusqu'alors si ceux-ci les réclamaient dans un délai de trois mois après leur arrivée. Enfin, le 12 décembre 1905, est promulgué un décret abolissant officiellement la traite des esclaves pour toute l'Afrique occidentale française. Or, différentes affaires présentes dans les rapports et la correspondance politique de l'administration civile coloniale de la région de Kayes de 1895 à 1906 montrent, dans la pratique, les aléas de cette politique, en particulier lorsque celle-ci est confrontée au statut ambigu des femmes esclaves.

7 On connaît encore peu de choses sur les stratégies d'émancipation des femmes esclaves en Afrique de l'Ouest au tournant du Xxe siècle. Cette question a été analysée plus en détail pour l'Afrique centrale et de l'Est notamment par Wright 1993. 
Ainsi, en octobre 1902, l'administrateur du cercle de Kayes apprend qu'une petite fille nommée Fatma, domestique chez une restauratrice européenne de Kayes, a été vendue par celle-ci à un Européen qui l'a lui-même revendue à un « indigène » de Kayes ${ }^{8}$. Lors de l'interrogatoire de police, la restauratrice explique que le propriétaire de la maison où elle a installé son restaurant lui a proposé, alors qu'il était sur le point de quitter la ville, de lui laisser la jeune Fatma pour une somme de deux cents francs. Par la suite, alors qu'elle se plaint régulièrement de Fatma, l'un de ses pensionnaires européens lui propose de l'en "débarrasser» pour cent cinquante francs. La restauratrice finit par accepter. Le pensionnaire déclare luimême qu'il a emmené la petite fille à la boutique du représentant de la maison Maurel $^{9}$ qui sert finalement d'intermédiaire pour la revendre à un certain Mallet, bourrelier à la direction d'artillerie de Kayes, pour la somme de trois cent francs. Le commissaire de police demandant à Mallet s'il ignore qu'il est défendu de vendre ou d'acheter des captifs, celui-ci répond « que ce n'était pas une captive qu'il entendait acheter, mais une femme pour son frère Boubakar, mécanicien à Saint-Louis, et employé au chemin de fer».

Cette affaire montre l'ambiguité de la condition des femmes esclaves dans les sociétés de la région. Ces femmes peuvent en effet être à la fois des biens d'échange, des travailleuses - ici en l'occurrence une domestique - et des concubines/épouses ${ }^{10}$. Fatma est revendue illégalement à deux reprises entre Européens comme un bien de rapport et, si la restauratrice ne semble pas faire de profit dans un premier temps puisqu'elle revend Fatma «à perte», son pensionnaire lui promet, s'il gagne quelque chose dans la vente, « de manger le bénéfice chez elle », ce qui fut fait. Mais c'est finalement un « indigène » qui rachète Fatma pour le compte d'un Wolof. Il cherche à se marier, mais « la chose était difficile car il n'avait aucune relation avec les gens de la ville ». Les protagonistes non européens savent

8 ANM Koulouba FA 1 E 201 : Correspondance affaires politiques du cercle de Kayes.

9 Maurel et Prom était une des maisons de commerce françaises les plus prospères en Afrique de l'Ouest au XIXe siècle.

10 Burrill $2008: 52$. 
probablement, malgré les dénégations de Mallet, qu'il s'agit d'une esclave. Les hommes dans la région de Kayes préféraient en effet parfois acheter de jeunes captives pour en faire plus tard leurs concubines ou leurs épouses, en particulier lorsqu'ils n'étaient pas assez riches pour avoir d'autres femmes ${ }^{11}$ ou lorsqu'ils n'avaient pas d'assise sociale et familiale suffisante dans la région. C'était souvent le cas des traitants et ouvriers wolofs ${ }^{12}$. L'intérêt, pour le maitre, était également que la captive n'avait pas de parents pour la protéger ou la reprendre en cas de problèmes ${ }^{13}$. Si elle refusait d'obéir à son maitre/mari, elle pouvait être vendue à nouveau ${ }^{14}$. Les guerres intestines qui ravagèrent l'Afrique de l'Ouest dans la deuxième moitié du XIX ${ }^{\text {e }}$ siècle, notamment les guerres de Samori dans le sud du Mali, produisirent un nombre considérable de captifs pour la traite interne ${ }^{15}$, multipliant ainsi pour les hommes libres et non libres les occasions d'acheter puis d'épouser des femmes esclaves ${ }^{16}$. Or, avec l'interdiction de la traite au Soudan, ce genre d'occasion devient de plus en plus rare, surtout dans une ville étroitement contrôlée comme celle de Kayes $^{17}$, où une affaire comme la vente de Fatma est rapidement découverte par les autorités.

11 Archives nationales du Sénégal (AS) Gouvernement général de l'AOF (GGAOF) FA 1 G 229 : Coutumier juridique du cercle de Kita, 1897. L'achat d'une captive est moins contraignant que les négociations de la dot qui peuvent durer plusieurs années avec l'obligation d'offrir régulièrement des cadeaux à la future bellefamille, cadeaux et dot dont le montant peut s'avérer au total bien supérieur au prix d'une jeune esclave. Le prix de la dot pouvait connaître, du fait d'autres prétendants, une inflation importante (Pollet \& Winter 1971 : 418-428).

12 Shereikis $2003: 222$.

13 Kopytoff \& Miers $1977: 32$.

14 Klein 2007a et ANM Koulouba FA 1 E 218 : Lettre n²7. L'adjoint des affaires indigènes, résident de la Haute-Gambie à l'administrateur du cercle de Bakel, 25 mai 1904.

15 Klein 1998 : 37-58; Hubbell 2001 : 27 ; Peterson 2005 : 4-6.

16 Une grande partie de ces esclaves furent vendu-e-s au Sénégal, et en particulier dans le bassin arachidier sénégambien, mais une partie non moins négligeable fut également absorbée par le marché local (Klein 1983 : 68-70), notamment dans la région de Kayes (Manchuelle 1989: 91) et sous forme de concubinage et de mariage (Rodet $2008: 171$ et $2010 \mathrm{a}: 281$ ).

17 La capitale de la colonie est transférée à Bamako en 1908. 
Cette affaire montre que l'administration ne comprend pas la condition ambiguë des femmes esclaves. Elle n'envisage pas que Mallet puisse être sincère quand il déclare que Fatma a été achetée pour devenir la femme de son frère. Mallet est finalement condamné à quinze jours de prison en attendant que le commandant de cercle reçoive des directives de la part de l'administration centrale. Quant aux protagonistes européens, ils doivent simplement rembourser les sommes gagnées lors des différentes reventes de Fatma. Malheureusement, les documents ne révèlent pas quelle fut la suite donnée à cette affaire ; il est possible que celle-ci ait été étouffée de peur qu'elle ne suscite un scandale en métropole.

On peut également, comme l'administration coloniale, envisager que l'acheteur wolof de Fatma, prétendant vouloir se marier, ait en réalité essayé de se procurer une jeune femme qu'il pourrait revendre à bon prix au Sénégal. En effet, la première interdiction de la traite en 1895 dans la région de Kayes fut peu appliquée par les successeurs de Grodet qui craignaient une perturbation des affaires économiques dans la région. La traite continua, notamment vers le Sénégal, sous couvert cependant de confiage, d'adoption et de mariage ${ }^{18}$.

Sur ce point, on peut rapprocher cette affaire de celle présentée en introduction. Dans cette dernière, Yackaré prétend que la jeune Gassa est sa fille alors qu'elle est son ancienne esclave, qu'elle a "mariée » à l'adjudant français et non vendue, afin de conserver ses droits de propriété sur elle et ses futurs enfants. Dans les sociétés de la région de Kayes, une femme esclave pouvait en effet devenir la concubine d'un esclave ou d'un homme libre. Elle restait cependant la propriété de son maître, de même que les enfants issus de l'union, à moins qu'elle n'ait été précédemment rachetée par l'homme qui désirait l'épouser, étant dès lors considérée comme libre. Le propre des femmes esclaves est que leur corps ne leur appartient pas et que leur

18 Rodet 2009a. L'exportation d'esclaves de la région de Kayes notamment vers Saint-Louis par le fleuve peut s'avérer une opération plus périlleuse car l'administration refuse de fermer complètement les yeux sur ce trafic et impose que les esclaves du Soudan français destine-e-s à l'exportation soient d'abord déclaré-e-s auprès de ses services qui leur délivrent automatiquement un certificat de liberté, ce qui peut donner l'occasion aux esclaves conscient-e-s de l'avantage de l'obtention d'un tel certificat de se rebeller contre leurs trafiquants. 
propriétaire a un droit sexuel absolu sur elles ${ }^{19}$. Elles sont donc régulièrement exploitées sexuellement. Ces faits restent cependant peu documentés pour la période coloniale, si ce n'est à travers quelques plaintes concernant des militaires. Dans l'affaire Gassa, on peut considérer le «mariage» avec l'adjudant pour la somme de 40 francs comme une vente du droit sexuel du propriétaire sur son esclave à un tiers. Yackaré sait certainement que ces concubinages ne durent que le temps du service de l'Européen dans la colonie. Cette vente est donc temporaire. Ce type de contrat revient finalement à une forme de prostitution puisqu'il y a clairement vente de services sexuels ${ }^{20}$.

Francis Simonis montre que "prendre mousso $»^{21}$ est une pratique largement tolérée voire encouragée par la hiérarchie militaire et administrative jusqu'en 1906, tant qu'elle ne porte pas atteinte à l'ordre colonial ${ }^{22}$. Le Docteur Barot écrit dans son Guide pratique de l'Européen en Afrique occidentale ${ }^{23}$ :

Pour ceux qui n'ont pas la force morale nécessaire pour supporter deux ans de continence absolue, il n'y a qu'une ligne de conduite possible: c'est l'union temporaire avec une femme indigène bien choisie.

19 Klein 2007a.

20 Si la prostitution d'esclaves par leurs propriétaires est bien connue pour les Antilles (Beckles 1989 : 141-151), cette question ne semble pas avoir été étudiée pour l'Afrique de l'Ouest.

21 White analyse également en détail la pratique du «mariage à la mode du pays » pour l'Afrique occidentale française, notamment à travers la question des métisses (White 2004 [1999] : 12-32).

22 Simonis 1993. Ceci expliquerait pourquoi les affaires traitées ici, malgré une tolérance générale pour le concubinage colonial, apparaissent dans les archives. Elles semblent en effet poser un problème aux autorités: elles ont été découvertes suite à des plaintes de la part de la population locale - mise à part l'affaire Fatma qui fait clairement scandale car elle met en cause des Européens. Or, l'administration est très attentive à ce qu'il n'y ait pas de friction avec la population locale alors que son pouvoir n'est pas encore complètement affirmé dans la région. C'est en partie d'ailleurs ce qui a retardé la mise en place d'une législation coloniale abolitionniste (Rodet 2009b : 271-273).

23 Barot 1902 : 328-330. Quoique publié en 1902, cet ouvrage s'appuie largement sur les expériences de militaires ayant participé à la conquête en Afrique de l'Ouest dans les deux décennies précédentes, notamment Desbordes et Meynier. 
Il argumente en soulignant que c'est une question de "sécurité sanitaire» qui évite aux coloniaux de fréquenter les prostituées «toutes contaminées», mais également une question de " considération » et de " discipline » qui permet, par un mariage avec la fille d'un chef influent, de faciliter l'administration de la colonie et de se faire respecter de ses subordonnés «indigènes ». Il s'agit enfin " d'hygiène » et " d'instruction », puisque la femme ainsi mariée prend soin physiquement et affectivement de son «mari», et lui fait partager sa culture. Ces concubinages sont donc encouragés tant qu'ils permettent une stabilisation à la fois de l'ordre politique et de la santé coloniale ${ }^{24}$. La "prise en charge» physique et affective n'est sans doute pas un vain mot puisque dans la réalité, ces relations de concubinage, tout en couvrant un vaste champ d'arrangements qui dépendent d'une équation économique, sociale et affective complexe, ressemblent finalement beaucoup aux relations entre maitres et esclaves dans les sociétés de la région où les femmes esclaves étaient surtout des domestiques offrant des commodités sexuelles.

Dans l'affaire de Gassa et Yackaré, l'adjudant semble profiter pleinement de la position ambiguë des femmes esclaves puisque, s'il donne dans un premier temps quarante francs à Yackaré pour ce «mariage », il finit, à la suite d'une dispute, par renvoyer Gassa et réclamer ses quarante francs. Mais, quelques jours plus tard, il reprend la jeune fille, à laquelle il verse cette fois directement vingt francs car elle a obtenu son certificat de liberté suite à leur «mariage ». L'administrateur, non dupe du caractère mercantile de l'affaire, conclue ainsi son rapport: «En résumé [il] a acheté pour la somme de $20 \mathrm{f}$ une jeune fille, a vécu avec elle deux ans en la nourrissant simplement!»

La conquête avait déjà donné lieu à de nombreux abus de la part des militaires qui s'appropriaient la plupart du temps femmes et esclaves selon le droit des vainqueurs ${ }^{25}$, mais certains de ces abus semblent s'être prolongés jusque dans les premières années de l'administration coloniale civile. Une autre affaire montre ainsi

\footnotetext{
24 Stoler 2002 : 48 et Lauro 2007 : 37-38.

25 Klein $1998: 83-84$ et 2007a ; Simonis $1993: 210$.
} 
comment les militaires continuèrent de profiter des facilités d'exploitation sexuelle des femmes esclaves de la région.

Fatimata, envoyée en 1902 par son maitre vendre du lait sur les chantiers du chemin de fer, y est retenue par des mesures de quarantaine consécutives à l'apparition de cas de fièvre jaune sur les chantiers de Boudofo ${ }^{26}$. Au bout de trois jours, elle est remarquée par un lieutenant qui la conserve comme concubine pendant huit jours. Lorsqu'il n'en veut plus, il la cède à son boy qui la revend ensuite à un officier d'administration du génie contre une somme de cent francs. Après trois jours avec cet officier, elle revient finalement vivre maritalement avec le boy du lieutenant qui souhaite désormais l'épouser en bonne et due forme. Cette affaire montre que des femmes peuvent être renvoyées du jour au lendemain, revendues, transmises à des subordonnés etc. En même temps, alors qu'elles sont prises dans des rapports de pouvoir qui bien souvent les dépassent, ces relations ne relèvent pas seulement de la domination, elles peuvent également dépendre de liens affectifs complexes ${ }^{27}$. Si les documents ne nous révèlent malheureusement pas les détails de ces relations, surtout du côté des femmes concernées, ils laissent cependant percevoir ce type de configurations affectives.

Ainsi, en 1906, le gérant européen du buffet de la gare de Toukoto adresse une lettre à l'administrateur de Kita pour lui demander la libération de deux captives, Goroko et Doualé, qui se sont réfugiées chez lui ${ }^{28}$. Après avoir engagé Goroko comme blanchisseuse, il réclame dans une seconde lettre à l'administration de lui confirmer que les deux femmes peuvent dorénavant être considérées comme libres $^{29}$. Il s'inquiète également du sort du jeune fils de Goroko qui a été repris par ses maitres. Une enquête administrative précise les circonstances de la fuite des deux femmes de chez leur maître : celuici les a envoyées à Toukoto pour y faire paître ses troupeaux; Doualé

26 ANM Koulouba FA 2 M 18 : Justice indigène. Correspondance cercle de Kita, 1887-1919.

27 Simonis $1993: 211$ et $2007: 66$.

28 ANM Koulouba FA 1 E 203 : Correspondance affaires politiques du cercle de Kita, 1883-1908.

29 AS GGAOF FA 15 G 151 : Kita, Correspondance arrivée, 1905-1906. 
serait devenue à Toukoto la maîtresse du gérant, qui aurait du coup encouragé les deux femmes à se rendre à Kita pour demander leur liberté. Les différentes tentatives faites par le gérant en l'espace de quelques semaines pour protéger cette famille et le ton de ses courriers à l'administration montrent l'attachement qu'il avait pour ces femmes, et plus particulièrement pour Doualé, sa maîtresse.

\section{La fin de l'esclavage :}

\section{une émancipation ambiguë en contexte colonial}

L'administration, voyant le lien intime qui semble unir le gérant et Doualé se montre finalement assez peu intéressée par cette affaire. L'administrateur de Kita refuse dans un premier temps de laisser Doualé retourner à Toukoto après qu'elle a obtenu sa liberté à Kita, probablement parce qu'il sait que Doualé est la maîtresse du gérant. En effet, l'administration, si elle tolère ces concubinages comme un mal nécessaire, n'en condamne pas moins leurs effets quand les protagonistes européens commencent à montrer de l'attachement pour leur concubine ${ }^{30}$. De plus, à partir de 1906, l'administration civile étant de plus en plus consolidée, les militaires quittant progressivement la colonie, on assiste à une stabilisation de la population européenne et à l'installation progressive de femmes et de familles françaises. Le concubinage est donc de moins en moins vu comme une nécessité par l'administration ${ }^{31}$. Les discours coloniaux condamnant les relations sexuelles et affectives interraciales commencent à apparaître alors que les femmes africaines sont clairement dévalorisées et racialisées ${ }^{32}$. Il s'agit de maintenir un ordre colonial qui trace clairement une frontière entre monde indigène et monde européen dans lequel la femme africaine représente l'ultime Autre de l'homme civilisé et universel ${ }^{33}$. La possession du corps des

\footnotetext{
30 Simonis $2007: 66$.

31 Simonis $1993: 214$.

32 Stoler 2002 : 51. Emmanuelle Saada a observé des discours coloniaux similaires quant aux relations interraciales en Indochine dans la première moitié du $\mathrm{XX}^{\mathrm{e}}$ siècle (Saada $2007: 56-58$ ).

33 Rodet 2009b : 27.
} 
Africaines par les colonisateurs est ainsi profondément inscrite dans la politique misogyne et raciste de la conquête ${ }^{34}$.

Le ton du courrier de l'enquête administrative dans l'affaire Doualé suggère que l'administrateur de Kita a décidé de déconsidérer totalement l'affaire car il ne voit sans doute en elle qu'une affaire sexuelle de plus entre un Européen et une Africaine qui ne mérite donc pas qu'on s'y attarde. En revanche, l'attention portée à l'enlèvement du fils de Doualé (l'administrateur parle de rapt d'enfant) montre que les autorités coloniales se soucient finalement bien plus des questions de traite - abolie quelques huit mois auparavant - que de l'émancipation de femmes qui, de toutes façons, arrivent à se libérer grâce à des relations intimes, notamment avec des Européens. La réaction de l'administration montre en réalité que lorsqu'il s'agit d'émancipation de femmes esclaves, elle considère celles-ci avant tout comme des concubines et des épouses, leur statut d'esclave n'étant que secondaire puisqu'elles doivent rester sous la tutelle d'un mari. Ce qui compte par contre beaucoup plus, c'est l'émancipation des hommes qui, du fait de l'abolition de l'esclavage et parce qu'ils sont bien souvent chefs de famille, doivent payer l'impôt et, de ce fait, entrer dans le monde colonial du travail pour trouver le numéraire nécessaire. Les documents coloniaux, puis les chercheurs, en ont conclu que l'émancipation était principalement masculine ${ }^{35}$. Or c'est sans doute davantage la politique coloniale d'émancipation qui fut masculine que la fin de l'esclavage elle-même qui a concerné en majorité les femmes ${ }^{36}$.

Par ailleurs, la condition des femmes esclaves de la région de Kayes et leurs relations spécifiques avec les Européens semblent avoir eu un impact plus général sur la vision coloniale des femmes au Soudan français. Dans les coutumiers juridiques, comme dans celui de Barat datant de 1899, les informations les plus précises sur les femmes concernent essentiellement l'esclavage. Cet état semble caractériser en grande partie la condition féminine. La femme est

\footnotetext{
34 Ce dernier aspect a été analysé en détail dans l'excellent ouvrage de McClintock (1995).

35 Paton \& Scully $2005: 1$.

36 Rodet 2008 et 2009 b.
} 
avant tout considérée comme une victime: elle est mariée et « héritée » contre son gré, elle est « domestique, productrice d'enfants, un objet de rapport estimé d'après son travail et le nombre de ses enfants ${ }^{37}$. L'étude de l'assujettissement des femmes indigènes permet aux Européens de réaffirmer la domination coloniale, conquérante et masculine, sur des sociétés jugées arriérées, passives, et donc féminines ${ }^{38}$. Les femmes soudanaises apparaissent également comme des objets fantasmés. Barat souligne que les femmes ne peuvent connaître un "amour idéal », mais seulement un "amour sensuel », « bestial ». Ce constat n'est pas étonnant si l'on a en tête les expériences sexuelles de ces femmes qui relevaient plus souvent du viol que de l'assentiment, que ce soit avec des hommes de la région ou des agents de la colonisation. L'auteur reconnait que la polygamie choque les Européens qui y voient des «scènes de harem » ${ }^{39}$. Mais il remarque également qu'après avoir précisément étudié le rôle de la femme dans le ménage polygame, la polygamie et l'excision paraissent être des pratiques justifiées dans le cadre soudanais car elles permettent de "réfréner leurs désirs sensuels ». L'auteur en déduit que la polygamie est une « nécessité naturelle et sociale $»^{40}$.

Le personnel administratif colonial s'appuie sur ces coutumiers pour "administrer au masculin $»^{41}$. Au quotidien, il est en contact principalement avec des femmes esclaves qui forment une large partie

37 AS GGAOF FA 1 G 229 : Coutumier juridique par Barat, Commis des affaires indigènes à Nioro, 1899. Les coutumiers compilent un certain nombre de pratiques observées par les Européens dans les sociétés locales et les érigent en « coutumes ».

38 Sibeud $2004: 192$.

39 Il est intéressant que Barat se réfère à la question du harem ici car si on a finalement peu d'informations sur la vie dans les harems ouest-africains, en particulier au Mali, Klein souligne que la majorité des femmes qui en faisaient partie étaient des esclaves (Klein 2007a et 2007b).

40 Quand on connaît les scandales liés à la polygamie affichée des militaires français pendant la conquête (Klein 1998 : 83), cette dernière remarque semble davantage relever de la justification détournée de telles pratiques que d'une réelle compréhension des habitudes locales.

41 Sibeud 2004 : 183. 
de la population de la ville de Kayes ${ }^{42}$. L'analyse des coutumiers est confortée à la fois par l'expérience qu'a le personnel administratif au quotidien de ces femmes esclaves et par sa propre représentation des rapports de genre en Europe. D'autres sources - les monographies des cercles de la région de Kayes au début du siècle - évoquent également les femmes, mais de manière superficielle, dans la catégorie «ethnographie». Ces textes livrent surtout des appréciations esthétiques ${ }^{43}$. La monographie du cercle de Kayes en 1904, de même que le coutumier de 1899, montrent que les administrateurs tentent de tirer de l'observation de quelques femmes des types sociologiques voire « raciologiques »; observations dont ils déduisent finalement le caractère général des populations: la femme rencontrée à Kayes, esclave ou ancienne esclave, ou lors de tournées administratives, devient «les femmes». Ils transforment une expérience personnelle en un texte ethnographique sensé faire autorité ${ }^{44}$. Cette vision misogyne et racialisée des femmes a encore, pendant longtemps, une influence directe sur le traitement par l'administration coloniale de la question du mariage et de l'émancipation des Africaines ${ }^{45}$. Cependant, les définitions coloniales mises en place à cette époque sont loin d'avoir dans la pratique la cohérence politique que le pouvoir colonial aurait sans doute souhaité, non seulement parce qu'elles s'adaptèrent aux circonstances et aux réalités de chaque région et de chaque colonie, mais aussi parce qu'elles furent régulièrement adoptées et contestées par les administré-e-s eux-mêmes.

Les affaires étudiées ici révèlent des voies d'émancipation qui ne s'accommodent pas vraiment des catégories coloniales. Même si elles sont rapportées par des administrateurs coloniaux qui utilisent leurs propres grilles d'analyse, une approche en termes de genre permet de détecter des stratégies spécifiques d'émancipation adoptées par certaines esclaves.

42 Rodet $2008: 174-175$.

43 Simonis souligne que la beauté était, pour les coloniaux, le critère de choix le plus important pour une concubine (1993 : 209-210).

44 Grosz-Ngaté 1988 : 485-486.

45 Rodet 2010b. 


\section{Relations de genre et stratégies d'émancipation}

Analyser ce type d'affaires est finalement assez frustrant car, si elles permettent d'imaginer les rapports de pouvoir qui pouvaient se jouer autour des relations intimes, il est difficile d'identifier les sentiments des femmes concernées. Ces documents sont en effet le produit d'un discours masculin dans lequel à aucun moment les voix des femmes ne sont entendues. Les seules sources qui permettent de les approcher sont les plaintes qu'elles ont déposées auprès de l'administration, ou encore les affaires de justice qui les concernent directement ${ }^{46}$. Si Catherine Quiminal a pu collecter quelques témoignages auprès de femmes âgées, concubines contraintes, ou de filles métisses sur leurs mères engagées dans des unions mixtes, de telles données restent rares ${ }^{47}$. Cependant, une approche de ces affaires qui prenne en compte les discours, les identités, les comportements et les relations de pouvoir associés à un sexe, de même que la construction sociale de la féminité et de la masculinitét8, permet de retrouver si ce n'est les voix des femmes, à tout le moins certaines de leurs stratégies d'émancipation. En effet, en lisant ces documents, il est important de se demander ce que cela pouvait signifier pour ces femmes d'être esclaves, quelles pouvaient être leurs options pour améliorer leur statut.

Même si l'administration s'avère plutôt réticente à promouvoir une réelle émancipation des femmes esclaves, notamment car celles-ci sont essentielles dans le processus de production dans des sociétés dont l'économie repose sur le travail servile ${ }^{49}$, cela ne décourage pas pour autant certaines esclaves de tenter de quitter leur maître quand elles en ont l'opportunité. Elles semblent notamment avoir réussi à utiliser les quelques voies ouvertes par le nouveau contexte abolitionniste. Malheureusement, on ne sait que très peu de choses de ces femmes, il est possible que leur jeunesse leur ait permis de

\footnotetext{
46 Klein \& Roberts 2005.

47 Quiminal 2008.

48 Vince, Rodet \& Goerg $2008: 3$.

49 La population esclave représentait environ $40 \%$ du total de la population de la région de Kayes, mais dans certaines zones elle pouvait atteindre jusqu'à $60 \%$ (Rodet 2009b : 277).
} 
s'attacher plus facilement à des agents de la conquête coloniale. Ces relations leur permirent sans doute de sécuriser une nouvelle source de protection et d'améliorer leur situation. Dans tous les cas, dans les sociétés patrilinéaires et virilocales de la région de Kayes, il n'est pas concevable pour une femme en âge de procréer de rester célibataire. «L'émancipation » signifie donc pour certaines captives la possibilité de choisir plus librement leur "protecteur». Les affaires étudiées ici permettent également de mieux comprendre dans quelle mesure ces stratégies risquent de remettre en cause des relations de genre et de pouvoir supposées fixes.

Dans l'affaire de Fatma, revendue à deux reprises, la jeune fille ne peut certes pas prévoir que ces ventes vont aboutir à son émancipation. Cependant, le témoignage de la restauratrice atteste indirectement de la capacité de Fatma à saisir des opportunités qui lui vaudront finalement sa libération. En effet, la restauratrice déclare qu'elle a demandé son accord à Fatma, avant de la céder à son pensionnaire. Si cette demande peut paraitre étonnante et peu crédible, elle n'est pas pour autant à mésestimer car si Fatma avait refusé de suivre le pensionnaire, la transaction n'aurait sans doute pas eu lieu. Pour échapper à cette vente, Fatma pouvait en effet se plaindre auprès de l'administration. Or, la restauratrice sait sans doute déjà que cette affaire est susceptible de faire scandale. Elle ne peut donc pas se permettre d'effectuer cette vente sans l'assentiment de Fatma. La restauratrice précise également «que si elle a voulu se débarrasser de cette enfant, c'est pour des raisons tout à fait intimes qui ne peuvent trouver leur place dans le présent rapport». Elle semble rejeter la responsabilité sur son concubin sans pour autant préciser les circonstances exactes de la vente. Il est perceptible d'après les différentes déclarations que Fatma n'était pas bien traitée par la restauratrice qui se plaignait d'elle en permanence et disait « qu'elle ne pouvait rien en faire et qu'elle ne serait jamais bonne à rien ». Les «raisons intimes » invoquées laissent à penser que Fatma posait non seulement un problème dans son travail mais également dans la vie privée de la restauratrice. Il est permis d'imaginer que le concubin de cette dernière ait eu des vues sur l'enfant. Fatma, mal traitée, peut-être abusée, consent finalement à sa propre vente, sans doute dans l'espoir que son nouveau maitre la traitera mieux. En effet, avant 
l'introduction de la législation coloniale abolitionniste, les esclaves de la région de Kayes non satisfait-e-s de la manière dont les traitait leur maitre tentaient souvent de trouver refuge et protection chez un autre. Certaines femmes esclaves essayaient également, pour améliorer leur situation, de trouver un nouveau «mari» avec une meilleure position économique et sociale. Les affaires Gassa, Dufort et ou encore celle des chantiers de Boudofo attestent de l'utilisation par les femmes de ces stratégies d'émancipation.

Dans l'affaire Gassa, celle-ci, après avoir été renvoyée par l'adjudant, accepte de revenir avec lui contre la somme de vingt francs. Lorsque celui-ci rentre en métropole, elle refuse de rejoindre son ancienne maîtresse qui, du coup, porte plainte auprès de l'administration. Gassa préfère en effet rester à Nioro chez un parent de sa maitresse que de retourner en condition de «non-libre » chez Yackaré. À Nioro, elle peut faire respecter son nouveau statut de non-libre du fait de la présence de l'administration coloniale. En même temps, son certificat de liberté ne lui permet pas d'échapper complètement à son ancien statut. Il lui est probablement difficile d'habiter seule et sans protection à l'heure où l'esclavage n'est pas encore officiellement aboli. Elle préfère donc réinvestir des liens d'allégeance passés, quitte à être considérée socialement comme une esclave, tout en ayant une plus grande marge de manœuvre puisque son certificat de liberté et la présence de l'administration coloniale à Nioro lui permettent d'éviter les abus liés à son statut.

L'affaire des chantiers de Boudofo montre également que les abus subis par les femmes esclaves ne les privent pas de la volonté de faire des choix qui peuvent les mener sur les voies de l'émancipation. Interrogée par l'administrateur de Kita, Fatimata refuse catégoriquement de retourner chez son maittre qui, déclare-t-elle, l'a toujours maltraitée. De plus, il ressort de l'enquête qu'elle ne reste finalement que trois jours avec le second officier. Elle prétexte du non versement de la somme promise pour revenir vivre maritalement avec le boy du lieutenant. Ce choix montre des préférences de la part de ces femmes pour certains hommes et donc pour certaines voies d'émancipation, notamment celles reconnues dans leur propre culture. Le boy désire en effet désormais épouser Fatimata et demande au commandant de Kita de servir d'intermédiaire pour 
proposer au maitre une somme de cent cinquante francs qui servirait de dot et de rachat.

L'affaire Dufort montre enfin les tentatives des femmes esclaves d'échapper à leur maître dès qu'elles en ont l'occasion en s'attachant à un agent de la colonisation. Le lien intime avec Dufort a non seulement permis à Doualé de garantir sa propre émancipation, mais également celle de sa mère et de son frère, de même qu'une position économique enviable localement, non seulement pour elle-même en tant que concubine de Dufort mais également pour sa mère désormais blanchisseuse pour celui-ci. Avec les débuts de la colonisation française dans la région de Kayes se sont donc multipliées les opportunités pour les femmes esclaves de s'émanciper, et cela même avant la fin officielle de l'esclavage en 1905. Les affaires étudiées ici sont riches d'informations sur la manière dont les femmes purent stratégiquement utiliser le concubinage et le mariage comme voies d'émancipation, voies qui finalement ne correspondent que peu à la représentation coloniale de l'esclavage, des femmes, du mariage et de la sexualité.

La fin du XIX siècle et les premières années du $\mathrm{XX}^{\mathrm{e}}$ siècle se révèlent une période charnière dans la fixation de certaines catégories genrées du discours colonial au Soudan français. Les archives montrent en effet que l'expérience coloniale de l'émancipation produit des discours spécifiques sur l'esclavage, le mariage, la sexualité et les femmes africaines dans des contextes de rapports de pouvoir et de violence dans lesquels les esclaves sont avant tout considérées comme des concubines, non concernées par la politique coloniale d'émancipation. Or, ces femmes adoptent au quotidien des stratégies qui montrent un champ complexe de rapports affectifs et d'allégeances s'accommodant mal des catégories coloniales. Du fait de la possibilité offerte à ces femmes de choisir leur concubin, une réelle émancipation se révèle finalement trop dangereuse pour les rapports de genre dans la région et risque donc de déstabiliser non seulement l'ordre social et économique, mais également l'ordre colonial qui compte s'appuyer sur la stabilité de «la famille africaine» pour affermir sa domination. Cette politique ambiguë de l'administration imprègne finalement la question de l'émancipation des femmes, comme celle du mariage, tout au long du XXe siècle. 


\section{Bibliographie}

BAROT Docteur, 1902, Guide pratique de l'Européen en Afrique occidentale, Paris, Flammarion.

BeCKLes Hilary, 1989, Natural Rebels: A Social History of Enslaved Black Women in Barbados, Londres, Zed Books.

BurriLl Emily S., 2008, "Wives of Circumstance": Gender and Slave Emancipation in Late Nineteenth-century Senegal», Slavery \& Abolition, 29/1, p. 49-64.

Capitaine Marceau du 134e régiment d'infanterie, 1911, Le Tirailleur Soudanais, Paris, Berger-Levrault Éditeurs.

DuPARC Camille, 2009, «Les femmes des tirailleurs sénégalais de 1857 à nos jours », Mémoire de Master II, Université du Havre.

ECHENBERG Myron J., 1980, «Les migrations militaires en Afrique occidentale française, 1900-1945 », Canadian Journal of African Studies, 14/3, p. 429-450.

Grosz-NgatÉ Maria, 1988, «Power and knowledge: The representation of the Mande world in the works of Park, Caillié, Monteil and Delafosse », Cabiers d'Études Africaines, 18/3-4, p. 485-511.

Hubbell Andrew, 2001, «A View of the Slave Trade from the Margin: Souroudougou in the Late Nineteenth-Century Slave Trade of the Niger Bend ", The Journal of African History, 42/1, p. 25-47.

Kanya-Forstner Andrew S., 1969, The Conquest of the Western Sudan, Cambridge, Cambridge University Press.

KLEIN Martin A., 1983, "Women in Slavery in the Western Sudan », in Claire C. Robertson \& Martin A. KleIn (eds), Women and Slavery in Africa, Madison, The University Press of Wisconsin, p. 67-92.

-, 1998, Slavery and Colonial Rule in French West Africa, Cambridge, Cambridge University Press.

—, 2007a, «Sexuality and Slavery in the Western Sudan », contribution présentée à la conférence internationale Sex, Power and Slavery: The Dynamics of Carnal Relations under Enslavement, McGill University, Montreal, 19-21 avril.

—, 2007b, "Sex, Power, and Family Life in the Harem. A Comparative Study », in Gwyn Campbeld, Joseph Miller \& Suzanne Miers (eds), Women and Slavery, Athens, Ohio University Press, p. 63-81.

KLeIN Martin A. \& Richard RoberTs, 2005, "Gender and Emancipation in French West Africa ", in Pamela SCully \& Diana PATON (eds), Gender and Slave Emancipation in the Atlantic World, Durham, Duke University Press, p. 162-180. 
KopytofF Igor \& Suzanne Miers, 1977, "African "Slavery" as an Institution of Marginality », in Suzanne Miers \& Igor KOPYTOFF (eds), Slavery in Africa. Historical and Anthropological Perspectives, Madison, The University of Wisconsin Press, p. 3-81.

LAuro Amandine, 2007, "Anxiétés, critique et offensives contre les ménagères et le concubinage interracial en situation coloniale : l'exemple du Congo belge (19081918) », in Odile Goerg (dir.), Perspectives historiques sur le genre en Afrique, Paris, L'Harmattan, p. 33-58.

MANCHUElLE François, 1989, «Slavery, Emancipation and Labour Migration in West Africa: The Case of the Soninke », The Journal of African History, 30/1, p. 89106.

ManN Gregory, 2006, Native Sons: West Africans and France in the Twentieth Century, Durham \& Londres, Duke University Press.

McClintock Anne, 1995, Imperial Leather: Race, Gender and Sexuality in the Colonial Contest, New York, Routledge.

PATON Diana \& Pamela SCully, 2005, «Introduction: Gender and Slave Emancipation in Comparative Perspective », in Pamela SCUlly \& Diana PATON (eds), Gender and Slave Emancipation in the Atlantic World, Durham \& Londres, Duke University Press, p. 1-34.

Peterson Brian, 2005, «Transforming the Village: Migration, Islam and Colonialism in French Southern Mali (West Africa), 1880-1960», Ph. D. Dissertation, Yale University, New Haven.

Pollet Eric \& Grace Winter, 1971, La société Soninke (Dyahunu, Mali), Bruxelles, Institut de Sociologie de l'Université de Bruxelles.

QUiminAL Catherine, 2008, « Du contrôle des femmes: unions, polygamie, sexualité », in Nancy L. GreEn \& Marie POInsot (dir.), Histoire de l'immigration et question coloniale en France, Paris, Cité nationale de l'histoire de l'immigration, La Documentation française, p. 239-245.

RodeT Marie, 2008, " Migrants in French Sudan: Gender Biases in the Historiography », in Toyin FAlOLA \& Niyi AfOlABI (eds), Trans-Atlantic Migration. The Paradoxes of Exile, New York, Routledge, p. 165-168.

- 2009a, "The End of Slavery and the Circulation of Juvenile and Female Workforce in the Region of Kayes, French Sudan (1900-1939) », contribution présentée au 11th Stanford-University of California Law and Colonialism Symposium, "Trafficking in Women and Children after the End of Slavery: Historical and Contemporary Perspectives from Africa and Beyond », 19-21 mars.

—, 2009b, Les migrantes ignorées du Haut-Sénégal (1900-1946), Paris, Karthala.

—, 2010a, «Mémoires de l'esclavage dans la région de Kayes: Histoire d'une disparition », Cahiers d'Études Africaines, 197, « Jeux de mémoires », p. 263-291. 
—, 2010b, «Continuum of Gendered Violence: The Colonial Invention of Female Desertion as a Customary Criminal Offense, French Soudan, 1900-1949 », in Emily Burrill, Richard Roberts \& Elizabeth ThOrnburry (eds), Domestic Violence and the Law in Africa. Historical and contemporary perspectives, Athens, Ohio University Press, p. 74-93.

SAADA Emmanuelle, 2007, Les Enfants de la colonie: les métis de l'empire français entre sujétion et citoyenneté, Paris, La Découverte.

SHEREIKIS Rebecca A., 2003, «Customized Courts: French Colonial Legal Institutions in Kayes, French Soudan, c. 1880- c. 1913 », Ph. D. Dissertation, Evanston, Northwestern University.

SIBEUD Emmanuelle, 2004, "Sciences de l'homme" coloniale ou science de "l'homme colonial"? Rapports de genre et ethnographie coloniale en Afrique française au début du XXe siècle ", in Anne HuGON (dir.), Histoire des femmes en situation coloniale. Afrique et Asie, XXe siècle, Paris, Karthala, p. 173-198.

SIMONIS Francis, 1993, "Splendeurs et misères des moussos. Les compagnes africaines des Européens du cercle de Ségou au Mali (1890-1962), in Catherine COQuery-VIDrovitch (dir.), Histoire africaine au XXe siècle. Sociétés-villes-cultures, Paris, L'Harmattan, Cabiers Afrique, 14-15, p. 189-206.

—, 2007, «Le commandant, ses compagnes, son épouse », in Odile GOERG (dir.), Perspectives historiques sur le genre en Afrique, Paris, L'Harmattan, p. 59-76.

Stoler Ann Laura, 2002, Carnal Knowledge and Imperial Power: Race and the Intimate in Colonial Rule, Berkeley, University of California Press.

Thompson, J. Malcolm, 1990, «Colonial Policy and the Family Life of Black Troops in French West Africa, 1817-1904 », International Journal of African Historical Studies, 23/3, p. 423-453.

TyMOwski Michal, 2000, «Les esclaves du commandant Quiquandon», Cabiers d'Études Africaines, 158/XL-2, p. 351-361.

VINCE Natalya, RodeT Marie \& Odile Goerg, 2007, «Introduction: Shifting Gendered and Colonial Spaces in Africa », Stichproben, 12, Fracturing Binarisms: Gender and Colonialisms in Africa, p. 1-11.

White Owen, 2004 [1999], Children of the French Empire: Miscegenation and Colonial Society in French West Africa, 1895-1960, Oxford, Oxford University Press.

Wright Marcia, 1993, Strategies of Slaves \& Women. Life-Stories from East/Central Africa, New York, Lilian Barber Press.

Zimmerman Sarah (à paraître), «Wives, Warriors, and Wards: West African Women Connected to the French Colonial Army, 1908-1918», International Journal of African Historical Studies. 